\title{
Analysis on the Energy Consumption and Carbon Emission of Jiangxi's Tourism Industry Using the Extended Kaya Identity
}

\author{
Junsong Jia ${ }^{1,2, a}$ \\ ${ }^{1}$ Key Laboratory of Poyang Lake Wetland and Watershed \\ Research, Ministry of Education, Jiangxi Normal \\ University, Nanchang, Jiangxi, 330022, China \\ ${ }^{2}$ School of Geography and Environment, Jiangxi Normal \\ University, Nanchang, Jiangxi, 330022, China \\ jiaaniu@126.com
}

\author{
Xiu Zhou ${ }^{1,2,3, b}$ \\ ${ }^{1}$ Key Laboratory of Poyang Lake Wetland and Watershed \\ Research, Ministry of Education, Jiangxi Normal \\ University, Nanchang, Jiangxi, 330022, China \\ ${ }^{2}$ School of Geography and Environment, Jiangxi Normal \\ University, Nanchang, Jiangxi, 330022, China \\ ${ }_{3}$ School of Graduate, Jiangxi Normal University, \\ Nanchang, Jiangxi, 330022, China \\ 2544931851@qq.com
}

\author{
Xinping Zhong ${ }^{1,2, c}$ \\ ${ }^{1}$ Key Laboratory of Poyang Lake Wetland and Watershed Research, Ministry of Education, \\ Jiangxi Normal University, \\ Nanchang, Jiangxi, 330022, China \\ ${ }^{2}$ College of City Construction, \\ Jiangxi Normal University, \\ Nanchang, Jiangxi, 330022, China \\ zxpdesign@163.com
}

\begin{abstract}
Firstly, we divide the energy consumption (EC) and carbon emission (CE) of Jiangxi's tourism industry into three groups and account them respectively. Then, we analyze the drivers of the total CE (Tc) by using the Arithmetic Mean Decomposition Index (AMDI) method based on the Kaya identity. Results show that the total EC (Te) is $6.78 \mathrm{PJ}$ in 2001, and it grows quickly up to $58.07 \mathrm{PJ}$ with an average annual increase rate of $19.6 \%$. Similarly, the Tc is $0.77 \mathrm{Mt}$ in 2001, and it increases to 6.58 $\mathrm{Mt}$ in 2013 with the same average annual increasing rate of $19.6 \%$. Especially in 2003, the Tc gets the least growth amount of $0.02 \mathrm{Mt}$ and increasing rate of $2.2 \%$ due to the Severe Acute Respiratory Syndromes (SARS) wreaks havoc. These mean people prefer to spend more and more time on the tourism activities from 2001 to 2013. However, the cumulative contribution of the population to the Tc's growth is the biggest one $(100.1 \%)$ and the other factor's contribution can almost be ignored. This denotes the major driving factor of the Tc's growth is the population itself. Therefore, to reduce the carbon emission of tourism, the consistent implementation of energy structure, energy-efficiency improvement policies, etc. is extremely needed in Jiangxi of China.
\end{abstract}

Keywords: tourism industry, carbon emission, Kaya, Arithmetic Mean Divisia Index (AMDI).

\section{INTRODUCTION}

The contribution of tourism to human-induced climate change is an increasingly important issue. However, the energy consumption (EC) and corresponding carbon emission $\left(\mathrm{CO}_{2}, \mathrm{CE}\right)$ of the tourism industry have never gotten enough attention. So, it is necessary to thoroughly discuss and analyze the $\mathrm{CE}$ and its drivers of the tourism industry nowadays.

In fact, a few scientists have made some certain achievements in this direction from several recent studies. For instance, this article [1] used an input-output framework to analyze the carbon embodied in both domestically produced and imported goods associated with tourism consumption. The article [2] used the life cycle assessment (LCA) to explore energy use and $\mathrm{CO}_{2}$ emissions of three islands' tourism and analyzed the primary contributors of the carbon emissions, respectively. The article [3] calculated the carbon dioxide emissions of Chengdu's tourism industry using the method introduced by IPCC report and found that the transportation was the major contributor.

All of these cases are not deep enough, and they often ignore the changes and the drivers of the total CE (Tc). Therefore, taking Jiangxi's tourism industry as an example, we, first, estimate the time-series' data of the EC and the CE, and analyze their change's trend.

Then, we use the Arithmetic Mean Divisia Index (AMDI) based on the Kaya identity to decompose the Tc's drivers. This has a certain innovative significance. The results gotten can help us find some good policysuggestions for regional low-carbon development, especially in the tourism industry. 


\section{DATA AND METHODS}

Data.

The original data sources of the EC and CE estimation in this study are from Jiangxi Statistics Yearbook, Chinese Tourism Statistics Yearbook and relevant bulletin from the Internet website of local tourism bureau, etc.; the parameters for estimating the tourism's EC/CE are from literature [4].

\section{Methods.}

The methods used for computing EC and CE of Jiangxi's tourism are from the article [4]. The formula of computing the tourism's EC/CE from the tourism [6] is:

$$
L E=\sum_{i=1}^{n} P \cdot l e_{i}
$$

where LE means the EC/CE of the tourism or its individual sector; $\mathrm{P}$ is the size of a specific tourism activity; lei stands for the EC/CE per unit from a specific tourism activity. In addition, total EC/CE of different vehicles can be denoted as:

$$
T E=\sum_{i=1}^{n} P_{i} \cdot D_{i} \cdot \beta_{i}
$$

where Pi means the total number of passengers taking transport mode $\mathrm{i}$; Di is the transport distance of transport mode i (km); ßi stands for EC or CE factor for transport mode $\mathrm{i}$. The AMDI used for decomposing driver is from the article [5]. The Kaya identity is from the [6]. But These two models are omitted for saving space.

\section{DYNAMICS OF THE EC AND THE CE FROM JIANGXI'S TOURISM}

Fig. 1 and Fig. 2 show the results of EC and CE arising from Jiangxi's tourism industry in 2001-2013, respectively, which are divided into three subcategories: tour traffic, lodging and activity.

For describing conveniently, we call them as Jt, Zs and Hd. So, Jte, Zse and Hde mean the EC of tour traffic, lodging and activity. Similarly, Jtc, Zsc and Hdc denote their CE, respectively. Table 1 denotes the shares of the CE's three subcategories in 2001-2013 and Table 2 indicates the increasing rate of the Jtc, Zsc, Hdc and Tc year by year from 2001 to 2013. In Table 2, the 01-02 means the increase situation of the CE in 2002 relative to the CE in 2001.

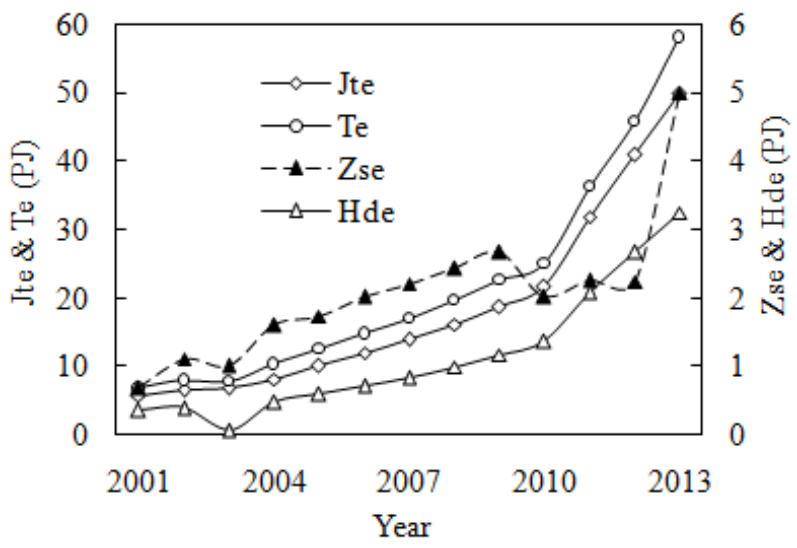

Fig. 1 EC of Jiangxi's tourism in 2001-2013

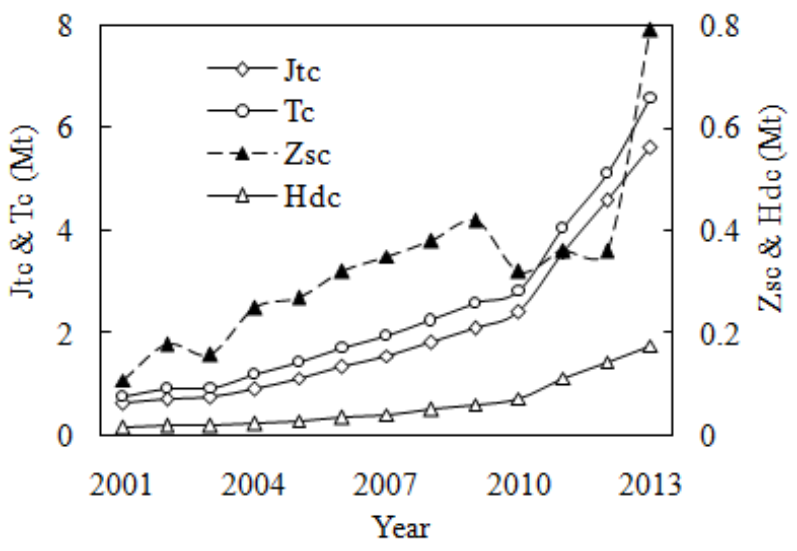

Fig. 2 CE of Jiangxi's tourism in 2001-2013

We can easily see the Tc of Jiangxi's tourism industry is $0.77 \mathrm{Mt}$ in 2001, and it grows quickly up to $6.58 \mathrm{Mt}$ in 2013 (Fig. 2). The growth quantity is $5.81 \mathrm{Mt}$, with an average annual growth rate of $19.6 \%$. Similarly, the Te of regional tourism industry is $6.78 \mathrm{PJ}$ in 2001 and it grows rapidly to $58.07 \mathrm{PJ}$ in 2013, with a growth's quantity of $51.29 \mathrm{PJ}$ and a same average annual growth rate of $19.6 \%$ (Fig. 1).

In fact, it can be obviously found that the change trend of the Tc is almost the same as the Te. This growth trend means people are willing to spend more and more time to engage in tourism-related recreational activities with the deepening of reform and opening up and the improvement of economic life in Jiangxi of China.

The Jtc is the largest share of the Tc, which always occupies about $80 \%$ of the Tc. The Jtc's share in 2004 is the least $77.0 \%$ and the share in 2012 is the most $90.1 \%$ (Table 1). Next to the Jtc, the Zsc's share is the second largest share of the Tc, which occupies about $18 \%$ of it. The Zsc's share in 2004 is the largest $20.9 \%$ and the share in 2012 is the least $7.1 \%$. The Hdc's share occupies about $2 \%$ of the Tc. The situation of the shares indicates the tour travel should be given more attention for its low-carbon development. 
The biggest instability of increasing rate is from the Zsc. The growth rate of it is $119.4 \%$ in 2013 , but the rate is $-23.8 \%$ in 2010 (Table 2). These may primarily be arising from the reform policy of the state's Star Hotel ratings in recently years.

However, Zsc's share and Hdc's are far less than the Jtc's (Table 1), the influence's effect of the first two categories' CE to the Tc can almost be ignored. So, the increase situation of the Jtc is almost the same as the Tc (Fig. 2). Similarly, the change trend, shares and increasing rate of the EC are almost the same as the CE (Fig. 1 and Fig. 2), but their analysis-related tables are omitted for saving space.

It can be easily seen the Tc speeds up obviously in 2004 (Fig.2 and Table 2). In 2003, the Tc's increasing rate is the least $2.2 \%$. However, it grows up to $27.7 \%$ in 2004 (Table 2). This may be arising from the influence of the ravages of Severe Acute Respiratory Syndromes (SARS) in 2003.

The Government has carried out some restrictions on people travels (including tourism) when the SARS wreaks havoc. While the SARS is under control in 2004, the restriction measures have also been released. Therefore, with the restriction released, the market demands of tourism industry have been freed too. So, the corresponding CE from Jiangxi's tourism industry has a significant growth.

Similarly, the growth rate of the Tc accelerates apparently in 2011 (Fig.2 and Table 2). In 2010, the Tc's increasing rate is $8.9 \%$. However, it grows up to $44.0 \%$ in 2011 (Table 2). This may be arising from the influence of the global financial crisis in 2008.

In 2008, China's government has launched a series of policies and measures to revitalize the economic development, including the four trillion's investment to the infrastructure construction, and so on. Correspondingly, people have also reduced much time to engage in tourism activities.

After two years of people's hard work, the world economy gradually embarked on a recovery track. Therefore, the market demands of tourism industry in Jiangxi of China have been freed again. So, the corresponding CE from Jiangxi's tourism industry has also a significant growth.

\section{DRIVERS ANALYSIS BY THE AMDI BASED ON THE KAYA IDENTITY}

Fig. 3 shows the decomposed factors' effect to the CE's growth. Fig. 4 denotes their corresponding contribution to Jiangxi's tourism CE. Table 1 and 2 indicate the cumulative effect and contribution of the decomposed factor from 2001 to 2013, respectively. The factors contain population (Pop), affluence per capita (Eco), energy intensity (Ene) and carbon emission coefficient (Car).

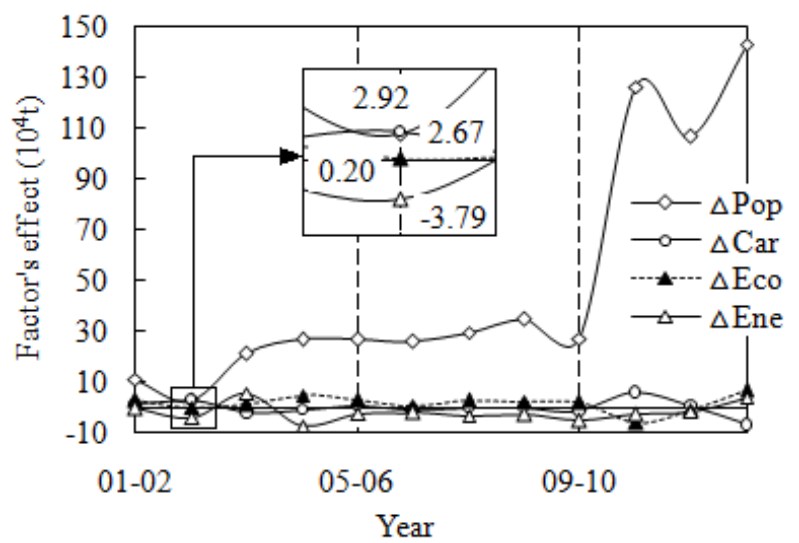

Fig. 3 Decomposed factor's effect to CE's growth

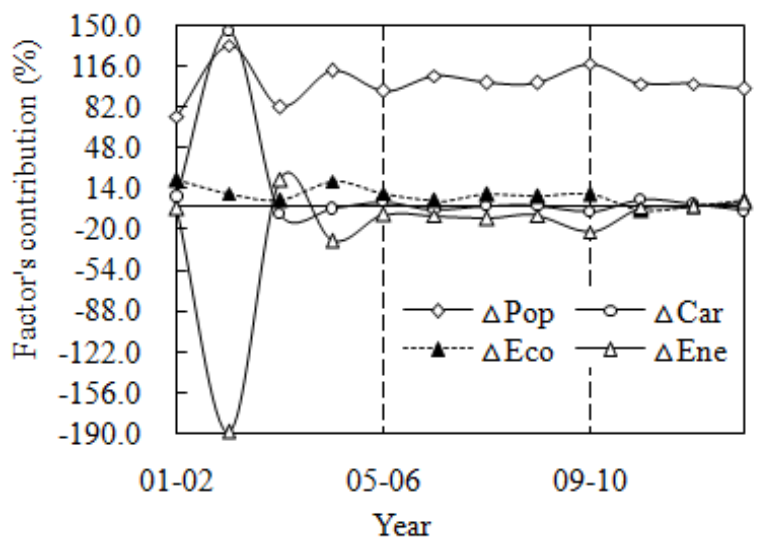

Fig. 4 The factor's contribution to CE's growth

From them, we can easily find the major driving factor of the CE's growth is the population effect and the other factor can almost be ignored. For instance, the Pop effect to the growth of the CE is $142.7 \times 104 t$ in 2013 . The Eco effect, Ene effect and Car effect upon the growth of the CE are 6.7, 4.0 and $-6.7 \times 104$ t, respectively (Fig. 3). Their contributions to the Tc's growth are $97.3 \%$, $4.6 \%$, $2.7 \%$ and $-4.6 \%$ in 2013 (Fig. 4).

The cumulative Pop effect, Eco effect, Ene effect and Car effect from 2001 to 2013 are 581.5, 20.3, -20.8 and $0.2 \times 104 \mathrm{t}$, respectively, and their cumulative contribution to the Tc's growth are $100.1 \%$, 3.5\%, $-3.6 \%$ and 0 (Table 1 and 2). So, we can conclude that the major driving factor of the CE's growth is the population itself. The affluence and technology's driving effect can almost be ignored because of their least contributions (100.1 >> 3.6, 3.5, 0).

However, as mentioned above, with the development of economics and improvement of living level, people prefer to spend more and more time on tourism-related travel or activities, and so on. Therefore, it is necessary for us to implement consistently the related policies of energy structure and energy-efficiency improvement so 
that reducing the carbon emission of tourism industry, especially in Jiangxi Province of China.

In 2003, with the SARS wreaks havoc, the Tc gets the least growth of $2 \times 104 \mathrm{t}(=0.02 \mathrm{Mt}$, Fig. 2$)$. However, the effects of four decomposed factors Pop, Eco, Ene and Car are 2.67, 0.20, -3.79 and $2.92 \times 104 \mathrm{t}$ (Fig. 3), respectively, which can just be added equal to $2 \times 104 \mathrm{t}$. The growth is so small that three absolute values of the factors' effects are largely more than the growth itself. So, three factors' contributions to the CE's growth show an obviously excessive (abnormal) situation in 2003 (Fig. 4).

In order to solve the causes or influences of this abnormal phenomenon, we could try using ridge regression, partial least squares (PLS) model, Hierarchical PLS (Hi_PLS) model, genetic programming, neural network, support vector machine and other nonlinear data model, etc., in the future. This is also the focus of our future research work.

TABLE 1 THE CUMULATIVE EFFECT OF THE DECOMPOSED FACTOR FROM 2001 TO 2013

\begin{tabular}{llllll}
\hline \multirow{2}{*}{ Year } & \multicolumn{5}{c}{ Cumulative factor's effect $\left(10^{4} \mathrm{t}\right)$} \\
\cline { 2 - 6 } & $\triangle$ Pop & $\triangle$ Eco & $\triangle$ Ene & $\triangle$ Car & $\triangle$ Tc \\
\hline $01-13$ & 581.5 & 20.3 & -20.8 & -0.2 & 580.7 \\
\hline
\end{tabular}

TABLE 2 THE CUMULATIVE OF THE DECOMPOSED FACTOR FROM 2001 TO 2013

\begin{tabular}{lcccc}
\hline \multirow{2}{*}{ Year } & \multicolumn{4}{c}{ Cumulative factor's contribution (\%) } \\
\cline { 2 - 5 } & $\triangle$ Pop & $\triangle$ Eco & $\triangle$ Ene & $\triangle$ Car \\
\hline $01-13$ & 100.1 & 3.5 & -3.6 & 0.0 \\
\hline
\end{tabular}

\section{CONCLUSIONS AND DISCUSSION}

So far, although many people have paid attention to the research of tourism's energy consumption and carbon emission, the existed studies are not deep enough, and they often ignore the trends and the drivers of the total carbon emission. So, taking Jiangxi's tourism as a case, we, first, estimate the time-series' data of the EC and the $\mathrm{CE}$, and analyze their change's trend. Then, we use some special models to analyze the total CE's drivers. This has a certain innovative significance.

The Tc of Jiangxi's tourism is $0.77 \mathrm{Mt}$ in 2001, and it grows up to $6.58 \mathrm{Mt}$ in 2013 with an average annual growth rate of $19.6 \%$. Similarly, the Te is $6.78 \mathrm{PJ}$ in 2001, and it grows to $58.07 \mathrm{PJ}$ in 2013 with almost the same growth rate. This means people prefer to spend increasing time on the tourism. In 2003, the Tc gets the least growth of $0.02 \mathrm{Mt}$ and increasing rate of $2.2 \%$ due to the SARS wreaks havoc. The Jtc is the largest share of the Tc, which occupies about $80 \%$ of it. This indicates the tour travel should be given more attention for its low-carbon development.

The drivers analyzed by the extended Kaya identity show the cumulative contributions of the Pop, Eco, Ene and Car effect are $100.1 \%, 3.5 \%,-3.6 \%$ and 0 , respectively. This denotes the major driving factor of the CE's growth is the population and the other driver's effect can almost be ignored. However, as mentioned above, people prefer to spend increasing time on tourism with the deepening of reform and opening up. Therefore, to reduce the carbon emission of tourism industry, the consistent implementation of energy structure, energy-efficiency improvement policies, etc. is extremely needed in Jiangxi of China.

\section{ACKNOWLEDGEMENTS}

The financial support of the National Natural Science Fund (71473113), Natural Science Foundation of Jiangxi (20132BAB213021), Scientific Research Foundation for Doctors of Jiangxi Normal University (4581), Scientific or Technological Research Project of Jiangxi's Education Department (GJJ14266) and Special Program of the Postdoctoral Science Foundation of China (201003158) are gratefully acknowledged. Corresponding author Junsong Jia can be contacted by (86)18607918843 or jiaaniu@126.com.

\section{REFERENCES}

[1] Munday Max, Turner Karen and Jones Calvin, Accounting for the carbon associated with regional tourism consumption, Tourism Management, 36, pp. 35-44, 2013.

[2] Kuo Nae-wen, Lin Chia-yun, Chen Pei-hun and Chen Yung-wei, An inventory of the energy use and carbon dioxide emissions from island tourism based on a life cycle assessment approach, Environmental Progress \& Sustainable energy, 31(3), pp. 459-465, 2012

[3] Liu Jun, Feng Ting-ting and Yang Xi, The energy requirements and carbon dioxide emissions of tourism industry of Western China, Renewable Sustainable Energy Reviews, 15(6), pp. 2887-2894, 2011.

[4] Wu Pu and Shi Pei-hua, An estimation of energy consumption and $\mathrm{CO}_{2}$ emissions in tourism sector of China, Journal of Geographical Sciences, 21(4), pp.733-745, 2011.

[5] Hatzigeorgiou Emmanouil, Polatidis Heracles and Haralambopoulos Dias, $\mathrm{CO}_{2}$ emissions in Greece for 1990-2002, Energy, 33(3), pp.492-499, 2008.

[6] Raupach Michael R, Marland Gregg, Ciais Philippe, Le Quere Corinne, Canadell Josep G, Klepper Gernot and Field Christopher $\mathrm{B}$, Global and regional drivers of accelerating $\mathrm{CO}_{2}$ emissions, Proceedings of the National Academy of Sciences of the United States of America, 104(24), pp. 10288-10293,2007. 